\title{
Chris Barker's Biological and Cultural Research in Development Theories of Physical Culture at Vietnam (In the Case of Nam Huynh Dao School)
}

\author{
Huynh Quoc Thang \\ Vietnam National University, Ho Chi Minh City, Vietnam
}

\begin{abstract}
In relation to human issues, the relationship between biology and culture has long been an important part of general social science research, cultural studies in particular. In which Chris Barker's theory of biology and culture in relation to related theories can be considered as one of the noteworthy remarks about the method and methodology to pass on the actual field Nam Huynh Dao school, the article focuses on clarifying the basic content of the problem with orientation to contribute to research and development of physical culture in Vietnam at present. This article was selected to read and print in the conference proceedings of scientific conference entitled "Theory of Western Culture and Applied to Vietnamese Culture Research" organized by the University of Social Sciences and Humanities, Vietnam National University-HCM City (USSH, VNU-HCM, Vietnam) (29 November 2018).
\end{abstract}

Keywords: biology and culture, physical culture, research to develop physical culture, school of Nam Huynh Dao

\section{Introduction}

Chris Barker is a researcher who has taught many universities in England and Australia, currently a professor in the Department of Arts, University of Wollongong, Australia. He has written many books on culture and media, especially related to issues of modern cultural life. In particular, the theory of biology and culture by Chris Barker is presented focusing on the topic "Biology and culture of the issues of treatise theory" (Biology, the body and culture, the problem of reductionism). This is one of 14 chapters of the "Cultural Study: Theory and Practice", a scientifically valuable project of interest to many readers around the world which has been reprinted many times. For the initial purpose of disseminating the problem in Vietnam widely, this article is mainly based on the translation of the work by Dang Tuyet Anh-Vietnam Institute of Culture and Art Culture, published in 2011 by the Culture and Information Publishing House (Hanoi) and it also was compared with the original text from the original, which was published for the third time and reprinted in 2008 (SAGE Publisher, California).

Huynh Quoc Thang, Ph.D., Associate Professor, Department of Cultural Studies, University of Social Sciences and Humanities, Vietnam National University, Ho Chi Minh City, Vietnam. 


\section{The Theory of Biology and Culture of Chris Barker With Research on Physical Culture Development and Some Features of the Nam Huynh Dao School}

\section{Go to Biological and Cultural Theory of Chris Barker}

The beginning of his thesis on "the relationship between human biology and human culture", C. Barker has affirmed that it is one of the "longest debate in the social sciences and humanity" (Barker, 2011, p. 161). Accordingly, "Reductionism" is mentioned by him as one of the methods and methodologies for analyzing and clarifying the "complexity" of biological problems in relation to culture from its most essential perspective. Like researcher D. Buss (Buss, 1999a; 1999b) once argued that the need to delete "binary pairs" includes: natural opposition to nurturing; culture versus biology; genetic contrast with the environment..., C. Barker has affirmed:

On the one hand, culture is a natural product of human learning and adaptation in the natural environment and ancestors. But, on the other hand, nature is not only a concept in language..., but the natural world is influenced by human knowledge and instincts. (Barker, 2011, p. 162)

C. Barker's research effort and results are aimed at "deconstructing" the opposition between nature and culture in the way: "Human culture and human biology both evolve and are indivisible separation", or "people are not only biological results born of nature but also cultivated by culture" and more "people are something that is stylized and has an attitude rather than a fixed and permanent physiological entity" (Barker, 2011, p. 164).

Based on the actual research, C. Barker has determined: "It is impossible to give absolute answers to requirements regarding the relationships between biology and culture" and "We cannot say what the relationship between biology and culture really is" (Barker, 2011, pp. 166, 168). However, he insists: "Human beings are a clear sign that humans are natural creatures" at the same time as "humans are not only biological results generated by nature but also refined by culture" and more "people are something that is stylized and has an attitude rather than a fixed and permanent physiological entity" (Barker, 2011, pp. 170-171). Most extensive, C. Barker said: "Our cultural elements are what we 'are'. Human consciousness itself is a product of cultural factors. Each of us can be described as a massive cultural element complex that runs on the physical apparatus of the human brain" (Barker, 2011, p. 195).

\section{About Physical Education and Research on Physical Culture Development}

The content of human research including physical culture issues, especially the interrelationships of human mental and physical processes, has been given a certain interest in historical reality of philosophical thinking, sociology, and many other angles ... That is more or less reflected in the analysis, system, explanation of the great ancient thinkers such as Socrat, Platon, Aristot, Hecralit ... until later, an important contribution of modern medical and scientific achievements, especially natural science, was built on the basis of biology, physics, and chemistry with great merit of Darwin, Newton, Enstein ... In it, the highlight is Darwin's theory of evolution and the theory of DNA-based reproduction and evolution that contributes to the explanation of "the evolutionary body of biology" in the same way as C. Barker wrote: "Evolutionary biology explores and explains the diversity of life on our planet, processes adapted by organic organizations to survive and explain the long-term development of species" or "Evolution is understood to be constituted by a stream of DNA flowing through time" (Barker, 2011, pp. 176-177). The problem posed in the way of explaining that it "is the process through which characteristics allow living and reproductive bodies to be passed down in a species" 
through "gene eyes" (about evolution) as R. Dawkins once mentioned (Dawkins, 1976). According to the same research directions, but further, J. LeDoux (LeDoux, 1998) argues that "Affection involves complex feedback mechanisms that exist between the brain, the body and their conscious experience. We, emotions are moving states" (Reiterated according to Barker, 2011, p. 186). Further look, S. Blackmore (1999) asserted "only when cultural elements are moved horizontally (across cultures) more often than vertically (from this generation through other generations) they become independent of the genes" (Reiterated according to Barker, 2011, p. 194).

Likewise, with their general thinking, countries in the traditional Asian culture area (including Vietnam) have also focused on applying the principles of the The Yijing; the center is the doctrine The Yin-Yang; The Five Basic Elements is seen as the quintessential achievement of philosophical thinking and is also the basis for all human physical activities, especially in medicine (oriental medicine), in martial arts in general.

Vietnam in the long history of the condition of geographical circumstances-equally harsh history has made significant achievements and accumulated on the treasure of knowledge related to military science (martial arts, experience beating foreign invaders...), traditional medicine, national martial arts... Up to now, although it has been initially interested in, the research on developing physical culture in Vietnam is still a common topic with many "white" areas, the researchers and scientific research results are still many limitations, not keeping up with the actual requirements, and are posing many serious problems. In addition to the strategy of Sports and Physical Training, The Overall Project of Physical Development and Stature of Vietnamese People in the Period of 2011-2030 was formally approved by the government with targeted programs and solutions such as: Research and development declare and apply key elements affecting the physical strength and stature of Vietnamese people; develop physical strength and stature with solutions to strengthen physical education for students from three years old to 18 years old; propagandize, educate, raise awareness, and change social behavior on physical development and stature of Vietnamese people... The national goal of human health in Vietnam in the next 20 years is to strive to improve. The average height of young people is $3 \mathrm{~cm}$ to 4 $\mathrm{cm}$; the average weight increases from $4 \mathrm{~kg}$ to $5 \mathrm{~kg} .$. (2011). All pose many pressing requirements for the cause of building people associated with the construction of Vietnam in the future, in which the orientation of research on physical culture development is definitely a big problem that cannot be particularly concerned.

\section{Go Back to Nam Huynh Dao With Activities Contributing to Physical Education and Researching for Development of Vietnamese Physical Culture}

One of the orientations of research is intimate and meaningful both in science and in practice that the current physical training and education is determined inseparably, but it is very necessary to attach immediately with the development and development of national culture, including national physical culture, in that there are national martial arts. This is one of the main reasons for the article to choose the object to be contacted in reality which is the Nam Huynh Dao School.

Nam Huynh Dao belongs to the Traditional Martial Arts Federation of Ho Chi Minh City, which is one of the traditional martial arts schools in Vietnam built and developed in the Southern region. The physical education activities of Nam Huynh Dao associated with building the spirit of martial arts in particular; the national culture in general is increasingly socially wide. It not only has a large impact on young people and old people, both male and female, from civil servants, workers, armed forces ... in urban areas to rural areas but also to the island, in particular, has initially built a martial arts school environment with the direct impact on 
millions of students in the school in some regions in both the North and the South... Moreover, Nam Huynh Dao joined and coordinated with the University of Social Sciences and Humanities, National University of Ho Chi Minh City organized a number of quite large scientific conferences about "Physical Education: Value, Reality and Solutions" and "Vietnam Martial Arts Culture" are the bases that have positive implications for contributing to research, saving contents, objectives, methods of physical education and development of Vietnamese physical culture in the current period.

\section{Research on the Development of Vietnamese Physical Culture on the Basis of Referring to the Theory of Biology and Culture of Chris Barker (Through Actual Contact of Nam Huynh Dao School)}

As a general rule, awareness for any object can go from narrow to the most meaningful or from cognitive meaning to practical meaning. Accordingly, the content of research on developing physical culture in Vietnam on the basis of referring to the theory of biology and culture of C. Barker (through actual contact of Nam Huynh Dao school) can be classified into: Physical With Physical Exercise and Sports, Physical With Living Culture, Physical With National Culture-Humanity.

\section{Physical With Physical Exercise and Sports (Biological and Cultural Relations in a Narrow Sense)}

With the viewpoint "The body is both a physical entity and a set of cultural symbols to classify, train and nurture people", C. Barker said: "Disease is not simply a consequence of the closed activities of individual bodies. Poor health is a consequence of what we eat, where we work (e.g. stress or chemical poisoning), levels and types of exercise..." (Barker, 2011, pp. 174-175) He also raised a more specific problem: "The transformation from biomedical to biomedical-psychology is marked by a change in language, from talking about "healing" to talking about "strengthen health"... from strengthening health to expanding the medical process to cultural organization and management of lifestyle ... Health promotion strategies are clearly based on the division of action into "good" and "bad" actions, but their management is our moral responsibility in the way "discipline produces internal force and internal force creates discipline" (Barker, 2011, pp. 175-176).

In fact, the Nam Huynh Dao school adopted its positive "therapies" to build a physical cultural foundation for thousands of disciples of all ages and subjects, including objects being addicted to drugs ... in order to step by step achieve physical well-being both physically and mentally, have a good attitude of life in all family and social relations, avoid social evils or bad habits (for example, most Nam Huynh Dao disciples do not smoke or quit cigarettes). It is not just an environment of constant physical training and martial arts (impact "body") but also an effort to build to create a martial arts cultural environment through learning activities, positive social activities ("mind" impact) ... With such initial practice, we can affirm that the physical is the "root" content of life with the core being the operation of the "body" and "mind" (here is understood as the basis of "physical/biological" and "substance/culture" relations in the lawful interaction of human movement with nature and society. Also through such a perception, it seems that all pose problems we need to redefine the goal and orientation of physical education in order to "thoroughly overcome the concept of awareness so far to see physical activity only" as a method of "physical training", "sports activities to win achievements" ... Although it may be practical indicators, if this is the case, then this is really a mental obstacle that inadvertently destroys all the essence of the true nature and physical life! This problem creates the thinking and the method of lack of accuracy is to turn physical into an object "tool" while in essence it is the "living religion", the "spiritual soul 
subject" of man. In other words, all forms of physical education, including sports, must be aimed at "promoting the true value of the spiritual and physical cultural life from 'tools' to 'purposes' really!” (Kiet, 2013, p. 170)

\section{Physical With Living Culture (Biological and Cultural Relations in a Broad Sense)}

As a person with deep research on modern culture and society, especially Western society, C. Barker stated a remarkable remark as follows: "Many of the key issues faced by Western cultures are psychological pain and not material deprivation". Such "pain" is often related to: human relationships (isolated, failed marriage, aggression and violence...); feeling the "nonsense" about life (hate, suicide, depression...); addiction status (alcohol, drugs, sex, shopping...); mental health (depression, anxiety...) (Barker, 2011, p. 183). The objectivity of the above judgment is from a deep perspective on the relationship between biology and culture on the basis of the reality of human life in the industrialization and modernization society as high as today. From there we can have explanations about its causes in many different directions. The most realistic explanation is that one can see the physical activity of the human being, which consists of two physical and mental levels in an interrelated relationship, in which

Spirit through mechanism of mind (consciousness) will be suppressed in the discrimination of judges, prejudices ... and the body is often "heated" by the "physical-level" liberation of physical laws which weakens and loses nervous system balance leads to physical dysfunction. All the more and more promote the process of depleting one's own vitality, making the division of human life force always be a permanent restriction and directly affect the negative direction all physical activities of people. (Kiet, 2013, p. 170)

In order to solve the reality with such sharp conflicts, physical education activities must contribute to promoting positive development of the value system in all forms of movement both at the physical and mental levels of people, all aiming to reach people in the following target directions: (1) regenerate the energy source of movement (when in operation), the basis of nourishment and improve life expectancy; (2) reasonable use of mobilizing energy sources in all interactions with living lifestyle, living environment, professional activities (due to positive effects of living morality, practice law...); contribute to positive all personal, collective, and community activities; (3) actively overcome the state of illness, bad habits, cultivate moral life, improve mental and physical capacity ... progress to the comprehensive development level of people with perfect cultural personality (Kiet, 2013, p. 173).

The above-mentioned points are essentially summaries of the initial experience through the practical experiences of Nam Huynh Dao according to the motto "Practice" as the key to focus on the goal of "Programming" with "code" is in combination with "Principles" to contact "Mind" (Spirit), outside then training "Gas" and building "Power" (Mechanical Strength) together with other self-conscious social behaviors to contribute to perfecting and building a Comprehensive Human Development on the foundation that "Culture itself" is increasingly formed solidly (Kiet \& Thang, 2013, p. 830).

\section{Physical With National Culture-Humanity (Biological and Cultural Relations in Practical Terms)}

In fact, biology and culture in relation to physical culture are all cultural entities associated with human and social cultural life, from a perspective of both contemporary and modern, on average personal and community, national and international representation. The concept of cultural life here can be defined to include all aspects of life (both material and spiritual) with cultural values contributing positively to creating quality and positive development for people and society, which mainly consists of Living Body (physical strength, intellectual strength, soul power), Living Relationship (with family, society, nature), Living Reason (dream, ideal, faith). 
These are different issues related to the areas of human life as stated as the content that must be constantly addressed in practice (Thang, 2017, p. 68). Through the practical activities of the Nam Huynh Dao sect as mentioned, we can prove that: "For adolescents, speaking of their physical strength is not only a complete development of muscles, nervous system, circulatory system ... but now it also has ... the relationship between health and mind wisdom, between physical strength and mental development... (Kiet, 2013, p. 70) On the basis of such a common, C. Barker considered the movement in biological and cultural relations all of which belong to the flow of "Evolutionary culture"; he said,

The cultural evolution view suggests that culture is formed as a legacy of previous adaptations to our ancestors' physical circumstances ... For example, every culture uses source symbols from the biochemical capabilities of the human brain ..., including forms of sexuality, family relationships, laughter, tears and rituals around birth, death and food... (Barker, 2011, p. 182).

The practical meaning of the problem is as C. Barker said that activities to develop human physical culture in general can be considered a part of the cultural life of the nation and expanded to be of all mankind. We can relate to the activities of Judo martial arts, Aikido ... of Japan, the Taekwondo of Korea and the traditional martial arts of Vietnam ... which have gradually become the traditional cultural values of the nation and of many countries around the world. These experiences can suggest many things not only for the preservation and promotion of the national martial arts heritage but also on the expansion of the scale of sports culture, strengthening the deep-root physical education measures more widely in the context of the current globalization, industrialization, and modernization.

Issues such as mentioned more significantly when relating to the actual operation as well as the orientation of Nam Huynh Dao's orientation to the physical education goals associated with "national cultural tradition" through construction "National Martial Arts". Looking deeply into the "Physicality of the nation", we can discover that it is the most realistic response, the highest satisfaction of human happiness needs associated with the national cultural life. In a more specific aspect, the Vietnamese nation's physical condition is always nurtured by a humanitarian life force associated with every process of struggle in the nation's history to create strong vitality that is full of spirit "Humanities-Martial arts" to meet the requirements of building and defending the Fatherland in all historical periods and to the extent that it has become a part of the common flow of human culture and to the extent that it has become a part of the general flow of human culture:

With the most common view, Vietnamese martial arts culture is firstly associated with the activities of traditional martial arts schools while focusing on preserving traditional values and towards a modern martial arts ... That cultural background brings spiritual strength while fostering the physical strength of a people who has the true happiness due to the Physics and Spirit that gives themselves through the "martial arts" tradition attached. The spirit of "Humanities" was formed very soon, right from the beginning... (Duong, 2017, pp. 17-18).

\section{Conclusions}

People are both a natural product and a product created by themselves that is culture. The biological and cultural relationship of $\mathrm{C}$. Barker and the related theories not only contribute to concretizing the above-mentioned scientific awareness but also can suggest many important issues for the practical application of life, especially in the current research and development of physical culture in Vietnam. In front of the requirements of the industrialization-modernization and international integration process, there has been a strong development, especially with the specific requirements and objectives of physical construction and 
development of Vietnamese people in new era; physical education work in a narrow sense is with sports, in a broad sense, with the culture of life and in the practical sense is with the cultural life of the nation-humanity... In fact, it is the content related to the different aspects of biological and cultural relations in awareness and reality, which are scientific-practical issues for the objectives and measures for physical education and research and development of physical culture in the current period. In the immediate future, through the research results of the theory of biological and cultural relations of C. Barker combined with the reality of the case of Nam Huynh Dao school, at least we have initially seen clearly that the study saving current Vietnamese physical culture as an important part of the strategy of building people is very necessary to continue to better apply all the achievements and results of research on the world in gender to proceed to build a system of scientific knowledge of national physical education increasingly improved and more effective.

\section{References}

Barker, C. (2008). Cultural studies: Theory and practice. Thousand Oaks, California: SAGE Publications.

Barker, C. (2011). Cultural studies: Theory and practice. (D. T. Anh, Trans.). Hanoi: Vietnam Institute of Culture and Arts-Information Culture Publishing House.

Blackmore, S. (1999). The meme machine. Oxford: Oxford University Press.

Buss, D. (1999a). Evolution psychology: The new science of the mind. Needham Heights, MA: Allyn \& Bacon.

Buss, D. (1999b). Evolutionary psychology: A new paradigm for psychology science. In D. Rosen and M. Luebbert (Eds.), Evolution of the psyche. Westport: Praeger.

Dawkins, R. (1976). The selfish gene. Oxford: Oxford University Press.

Duong, M. (2017). Commentary on a Vietnamese martial arts culture. In the proceedings of the Scientific Conference on "Vietnam Martial Arts Culture" (pp. 11-18). Ho Chi Minh City University of Social Sciences and Humanities-Nam Huynh Dao School held, Ho Chi Minh City.

Kiet, H. T. (2013). Physical culture and advocacy science. In the proceedings of Scientific Workshops "Physical Education: Value, Reality and Solutions” (pp. 166-177). Social Sciences and Humanities University of Ho Chi Minh City—Nam Huynh School Dao Organization, HCMC.

Kiet, H. T., \& Thang, H. Q. (2013). Vietnamese martial arts with Confucianism (in the case of Nam Huynh Dao). In the proceedings of the International Conference "Confucianism From the Perspective of Cross-Cultural" (pp. 726-931). University of Social Sciences and Humanities-VNU HCMC.

LeDoux, J. (1998). The emotional brain. London: Phoenix.

Prime Minister: Decision No. 641/QD-TTg. (April 28, 2011). The overall project of physical development and Vietnamese stature in the period of 2011-2030.

Thang, H. Q. (2017). Free time with building cultural life of teenagers. Human Development Magazine, 2(53), 66-74. 